\title{
Performance Evaluation of Position-based Routing Protocols using Different Mobility Models in MANET
}

\author{
Omar Almomani \\ Faculty of Information Technology \\ The World Islamic Sciences \& \\ Education University, Jordan
}

\author{
Mahmoud Al-shugran \\ Faculty of Information Technology \\ Jerash University, Jordan
}

\author{
Jafar A. Alzubi \\ Al-Balqa Applied \\ University \\ Al-Salt, Jordan
}

\author{
Omar A. Alzubi \\ Al-Balqa Applied \\ University \\ Al-Salt, Jordan
}

\begin{abstract}
A Mobile Ad hoc network (MANET) is a dynamic selforganizing multi-hop wireless network. Owing the random and unpredictable movement of the mobile nodes, the topology is changing rapidly and frequently. In MANET, where is no routing infrastructure like fixed routers and routing backbones, all MNs participate in the routing process. Recently, position-aware routing protocols, and because of their simplicity, position awareness, and scalability, are the most routing protocols used with MANET. In recent years, a variety of position-aware routing protocols have been developed. The efficiency of such protocol affects by the used underlying mobility model. Thus, wrong selection of the Mobility model may has devastating consequences on the performance MANET. Research efforts haven't focused much in evaluating their performance when applied to different mobility models. In this paper, we have studied the effect of two different mobility models; Random Waypoint mobility model (RWP) and Boundless Mobility Model (BDM) on the performance of selected group of position-based routing protocols. The performance analysis was carried out by using the network simulator, Ns2. The simulation results clarify the performance of the selected routing protocols with three performance metrics.
\end{abstract}

\section{Keywords}

MANET, Routing Protocol, Mobility Model, RWP, BDM.

\section{INTRODUCTION}

Mobile ad hoc network (MANET) is a self-organized network; it consists of mobile nodes in the fly. The mobile nodes communicate wirelessly, and can serve as both hosts and routers at the same time [1]. MANET is a multi-hop wireless networks where all participating nodes involved in the routing process. Hence, a routing protocol in MANET runs on every node and is affected by the resources at each mobile node [2].

MANET performance and reliability depends on the used routing protocol [1]. Such routing protocols need to work well not just with a law mobility and small network. But also, we need more dynamic routing protocol that responds quickly to high mobility, and frequent topology changes. Recently, position-aware routing protocols, and because of their simplicity, position awareness, scalability, are the most used with MANET.

The mobility characteristics of mobile nodes represent the changing over time in their speed, direction, and acceleration values [3]. In the simulation environment, the Mobility models mimic the movement pattern of the participating nodes for any MANET protocol [4]. To evaluate the performance of any new routing protocol, the selected mobility model to be used with this protocol has a highly consideration [5]. Thus, the selected mobility model may have negative effects on the position-based routing protocol and can harm its performance [6].

The goal of this paper is to analyze the performance of different position-based routing protocols under two different mobility models. The selected routing protocols are; Greedy Routing protocol (GFS) [7], Greedy Perimeter Stateless Routing (GPSR) protocol [8], Directional Greedy Routing Protocol (DGRP) [9], and Mobility-based Adaptive Greedy Forwarding (MAGF) [10]. The performance evaluation has been done with two types of mobility models which are Random Waypoint Mobility Model RWP [11], and Boundless Mobility Model BDM [12]. In this paper, the comparison held in two different scenarios, each performed under three selected performance metric.

\section{RELATED WORK}

Mobility pattern, in many previous studies was assumed to be random waypoint. More recently several studies in the state of the art argued that this model have served several drawbacks in the performance of routing protocols. The difference of results is spectacular, and it is clear that such a conflict has devastating consequences on the performance of routing protocol and MANET performance.

Bettstetter and Krause in [3] have shown through simulation results that the RWP model introduces a non-uniform distribution of mobile nodes location. Furthermore, the results gathered in [3] has been analytically confirmed by Chu and Nikolaidis in [13], where it was shown that using random waypoint model incurs hot spot problem at the center of the simulated network. Divecha et al. in [14], have shown that in the case that density is directly proportional to the throughput of the MANET network, this result is acceptable if the density does not acceded a certain level where the performance of the routing protocol will be decreased and degraded.

Camp, et al. in [6] has shown that classic Random Waypoint is unsuitable to mimic the Features of the real-world nodes' mobility patterns. Also they argued that RWP model can generate unreliable results.

Harri et al. in [15] argued that the main challenge posed by the wireless network is the characterization of network mobility level. The Random Waypoint model, which is the well known model, generated of random linear speed-constant movements within the simulated area boundaries. Also, they 
have shown that using any Random Waypoint model produces useless and unreliable results that affect the performance level. This comes as a result of higher density of mobile nodes in the center of the simulated area. Yan et al. in [16] proved experimentally that the hired mobility model in MANETs may greatly affect network performance in terms of throughput, loss, and delay.

\section{OVERVIEW OF SELECTED ROUTING PROTOCOLS}

In this section, we briefly describe the key features of the unicast position-based routing protocols studied in our simulations. Generally, greedy forwarding strategy (GFS) considered the most used routing protocol in MANET. Regarding to the dead-end handling procedures, the proposed position-based routing protocols can be broadly divided into two main categories. These categories are; Recovery Strategies to Handle GFS Failure (RSGF), and Supportive Enhancement for GFS (SEGF). In RSGF, once GFS fails due to dead-end problem, the recovery phase should be executed to continue routing the stuck packet around the void. With SEGF, GFS is enhanced by adopting other metrics besides distance metric to achieve other objectives besides shortest path objective. With SEGF, the proposed solution is still in need to the recovery mode to be executed as GFS fails due to dead-end problem.

To cover each category in our comparison we select Greedy Perimeter Stateless Routing (GPSR) from RSGF category. Further, from SEGF category we select Directional Greedy Routing Protocol (DGRP), and Mobility-based Adaptive Greedy Forwarding (MAGF). The common thing with all above protocols, that each of them uses greedy routing (GFS) as initial forwarding mode. To elaborately purpose of this work, we also add the conventional position-based routing protocol GFS.

With GFS, each node selects the next relay node as the one closest to ultimate target than itself. The packet continues forwarded greedily till it ends in a void node. Thus, we can say that our greedy routing is fail, and one of the recovery modes should continue the forwarding mission in behalf of GFS. In this work we denoted GFS as GPSR* (GPSR version with perimeter mode switched off).

\section{MOBLITY MODELS OF MANET}

Mobility model mimics the movement of the mobile nodes within the network [18-20]. Thus, and as proved in [21], the selected mobility model for simulation can impact the proposed protocol performance significantly. Also, the works in [22-24] emphasize the same idea, and they argued that selected mobility model affects MANETs in multiple aspects, such as; routing performance and network capacity.

Based on the results presented in [21-24], the authors proved that the selected mobility pattern is considered as a selective issue, and it should accurately characterize the planned scenario in which the proposed protocol is likely to be used. Moreover, in order to derive performance bounds and to understand the limitations of our proposed algorithm in comparing it with other work, such mobility model should be mathematically tractable. Finally, the selected mobility model should be flexible enough to furnish, qualitatively and quantitatively, various mobility features when varying some parameters of the selected model.

\section{ANALYSIS OF SELECTED ENTITY MOBILITY MODELS}

In this section, we only briefly introduce the two compared entity mobility models RWP, and BDM. For full extra information about mobility models the work in [4] can be concern.

\subsection{Random Waypoint Mobility Model}

Random waypoint is a simple model that is easy to analyze and implement. This has probably been the main reason for the wide spread use of this model for simulations. The Random Waypoint Mobility Model has been used for the first time in [11]. In the current network simulator (NS-2) distribution, the implementation of this mobility model is as follows: each mobile node in the simulation area of fixed size, assigned an initial self-position, and a destination position. The two positions are selected independently and uniformly on the simulation area. The speed of MNs is chosen uniformly on an interval [Vmin, Vmax], independently of both the initial self-position and destination's position. Next, MN starts to travel from its current position towards the selected destination with constant velocity chosen uniformly and randomly from The pause time is selected uniformly between 0 and pre-specified period of time independently of speed and positions. After reaching the destination, a mobile node starts its staying in the destination for a certain pause time. Once the pause time expires, the $\mathrm{MN}$ selects a new random destination and new speed independently of all previous destinations and speeds. It then starts traveling towards the new selected destination at new speed.

\subsection{Boundless Simulation Area}

Completely contrary to Random waypoint model, the movement of mobile nodes with the Boundless Mobility Model interlaces and considers the relationship between the previous movement characteristics of the mobile node with its current characteristics [12]. In BDM model, velocity and motion direction of current movement randomly diverge from the previous velocity and motion direction after each time increment. This introduces a smooth node's movement in both velocity and motion direction. The position, velocity and motion direction are updated at every $(\Delta \mathrm{t})$ time steps.

The most advantage of using BDM is refer to its unique behavior as a mobile node reaching the border of the simulation area it does not return back into simulation area; instead it continues to go forward and reappears on the other side of the simulation area. Thus, this advantage removes the negative side effects of the simulation edge in the performance evaluation of the used routing protocol. As an outset of the unique BDM's characteristics, it creates more realistic node movement traces.

\section{SIMULATION STUDY}

Below there is a description of the simulation and mobility model set-up. The influence of the two selected mobility models on the performance of position-based routing protocols is stated.

\subsection{Simulation Set-up}

The goal of the simulation is to investigate the performance of the selected routing protocols based on the two mobility models, we use network simulator 2 version 2.34 [17]. For this simulation sake, we use the default settings for the GPSR, PDGR, MAGF, and GFS routing protocols and with the following specifications; 
- The simulation network area is $2500 \mathrm{~m} \mathrm{x} 2000 \mathrm{~m}$ rectangle with $250 \mathrm{~m}$ nodes' transmission range. We use the MAC layer protocol 802.11 DCF RTS/CTS. Bandwidth (Bw) set to standard value of 4 mbps. Traffic model uses Continuous Bit Rate (CBR) traffic sources. Traffic sources transmit data at a fixed data rate of 5 packets/s. Data packet size set to standard values 512 bytes and beacon packet size is 64 bytes. We consider randomly 15 source-destination pairs.

- To get perfect results through performing the simulation, the experiments were executed in two different scenarios.

$>$ In the first scenario, the two mobility models are compared in different number of nodes; 50, $100,200,300,400$, and 500, and with fixed speed $20 \mathrm{~m} / \mathrm{s}$.

$>$ In the second scenario, the two mobility models are compared in different speed values; $5,10,20,30$ and $40 \mathrm{~m} / \mathrm{s}$, and with fixed the number of node to 200 nodes.

- All the performance results presented are an average of 50 different simulation trials. The simulation for each scenario is executed in a period of 1200 , seconds, and to avoid the effect of initializing and ending, we only gather the data between $800 \mathrm{~s}-1000 \mathrm{~s}$.

\subsection{Mobility Models Set-up}

Table 1 summarizes the main simulation parameters and their values used in this paper that is relevant to Random Waypoint Mobility and Boundless Mobility Models.

Table1. Simulation parameters of Random Waypoint Mobility and Boundless Mobility models

\begin{tabular}{|c|l|l|l|}
\hline Waypoint & Description & Value & Unit \\
\cline { 2 - 4 } Mobility Model & Pause time & 0 & $\mathrm{~s}$ \\
\cline { 2 - 4 } & Speed interval & {$[1,40]$} & $\mathrm{m} / \mathrm{s}$ \\
\cline { 2 - 4 } & Motion direction & {$[0,2 \pi]$} & degree \\
\hline
\end{tabular}

\begin{tabular}{|c|l|l|l|}
\hline Boundless & Description & Value & Unit \\
\cline { 2 - 4 } Mobility Model & Maximum speed $\left(v_{\max }\right)$ & 40 & $\mathrm{~m} / \mathrm{s}$ \\
\cline { 2 - 5 } & Maximum acceleration $\left(A_{\max }\right)$ & 10 & $\mathrm{~m} / \mathrm{s}^{2}$ \\
\cline { 2 - 5 } & Maximum angular $(\alpha)$ & 90 & degree \\
\cline { 2 - 5 } & Updating time steps $(\Delta t)$ & 1000 & $\mathrm{~ms}$ \\
\hline
\end{tabular}

\section{PERFORMANCE EVALUATION METRICS}

For the simulation results, and to compare the two mobility models, we consider the following performance metrics:

- $\quad$ Packet Delivery Ratio (PDR): This ratio represents the number of data packets successfully received by the destination to the total number of packets sent to destination.

- $\quad$ End-To-End Delay (E2E-D): This metric represents the difference between the time a data packet is received by the destination and the time the data packet is generated by the source.

- Routing overhead: This metric represents the ratio between the numbers of control packets transmitted for every data packet sent. And we used the following formula to find it out.

\section{EXPERIMENTAL RESULTS}

This section presents the results of the conducted experiments to analyze and compare the impact of Random Way Point and Boundless Mobility Models on position-based routing protocols. The cropped results are based on the two scenarios that have been discussed in the previous section to show the effects of each mobility model.

\subsection{Performance of Selected Routing Protocols for Varying Number of Nodes}

In the first scenario, the speed of MNs is fixed to $20 \mathrm{~m} / \mathrm{s}$ and compared with variance number of participating nodes; 50, 100, 200, 300, 400, and 500. RWP and BDM mobility models were tested on selected routing protocols. To finalize the results, this work based on three performances metric which are Packet Delivery Ratio, End-to-end delay, and Routing Overhead.

In our simulation for varying number of nodes we can notice that performance of selected routing protocols using BDM is much better than RWP. Figures 1 up to Figure 12, depicts in details the effects of both mobility models on the performance of the selected routing protocols. From the results it is clear that through employing very low number of nodes that using RWP mobility model negatively affected the performance of the selected routing protocols compared when using BDM mobility model. Because the high performance of RWP much related to the density of nodes at the center of simulation area, and with high distributed nodes with no movement correlation this defiantly harms RWP performance. As the number of nodes is increased the performance becomes more or less constant with both mobility models, but if density is too large, more and more of nodes try to access the common medium, which results in more congestion at the interface of the mobile nodes and the number of collisions increase. This can severely degrade the performance of both mobility models that will affect the performance of selected routing protocols in terms of decreasing packet delivery ratio and increasing overhead and show more delay. Owing the hot-spot area and uncorrelated motion problems of RWP mobility model, using BDM mobility model result in high performance even with dense networks. As a result, the performance of the selected routing protocols shows noticeable dependence on the selected mobility model. BDM mobility model outperforms RWP mobility model in both sparse and dense networks. Thus the performance of position-based routing protocol using BDM mobility model improved too.

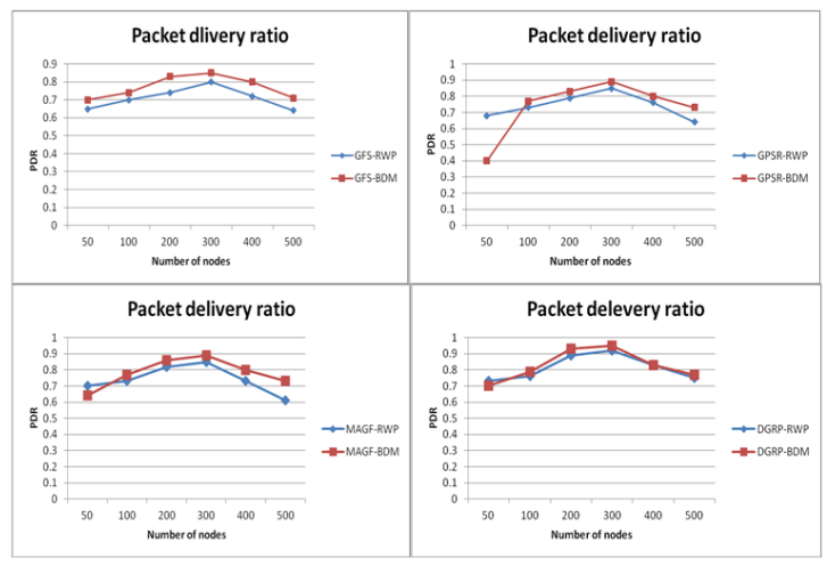

Figs 1-4: Packet delivery ration with the selected routing protocols vs. both mobility models using various nodes number 


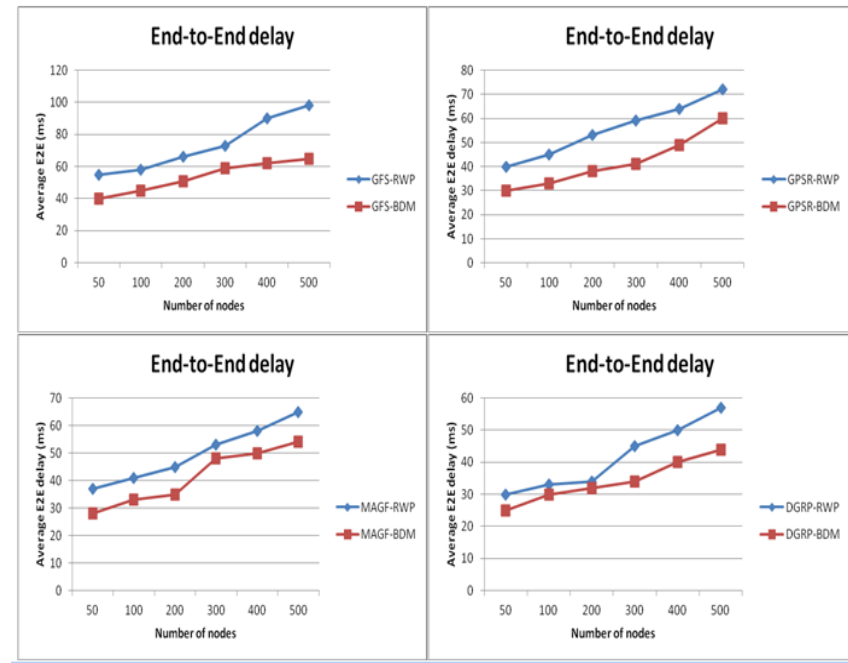

Figs 5-8: End-to-end delay ration with the selected routing protocols vs. both mobility models using various nodes number

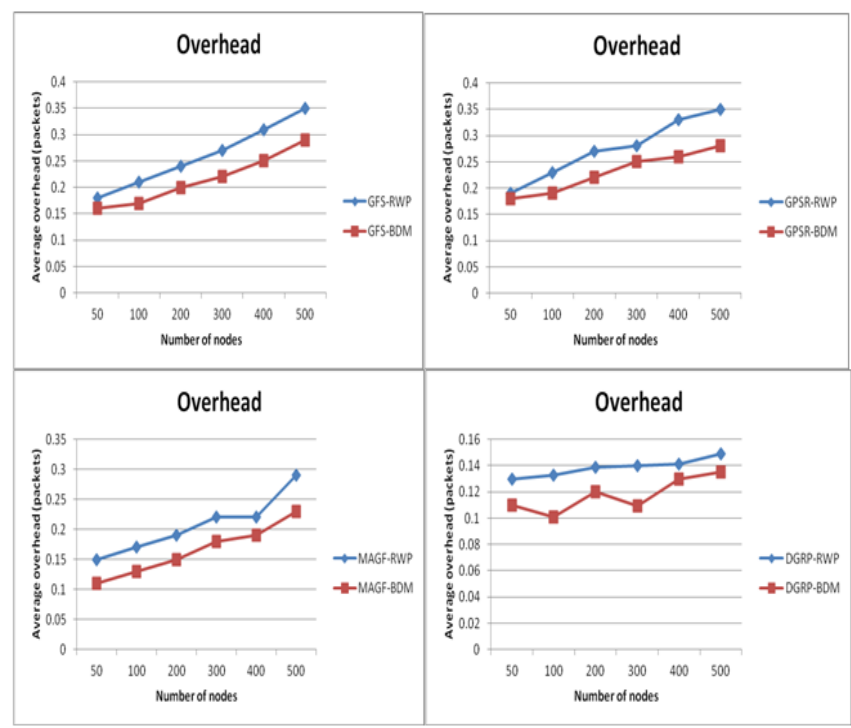

Figs 9-12: Overhead ration with the selected routing protocols vs. both mobility models using various nodes number

\subsection{Performance of selected routing protocols for varying speed on both mobility models}

Due to the significant aspect of speed in the used mobility model, this scenario, deployed fixed number of participating nodes which is 200 nodes, and varies the movement speed as; $5,10,20,30$ and $40 \mathrm{~m} / \mathrm{s}$. With these two specifications, the two mobility models were tested on the selected positionbased routing protocols. The total throughput of the system was averaged. As illustrated in Figure 13 to Figure 24 it is clear that as the mobility increases; the performance of both mobility models deteriorates. But in all cases, BDM performs better than RWP, Which proportional to the performance of the selected routing protocols.

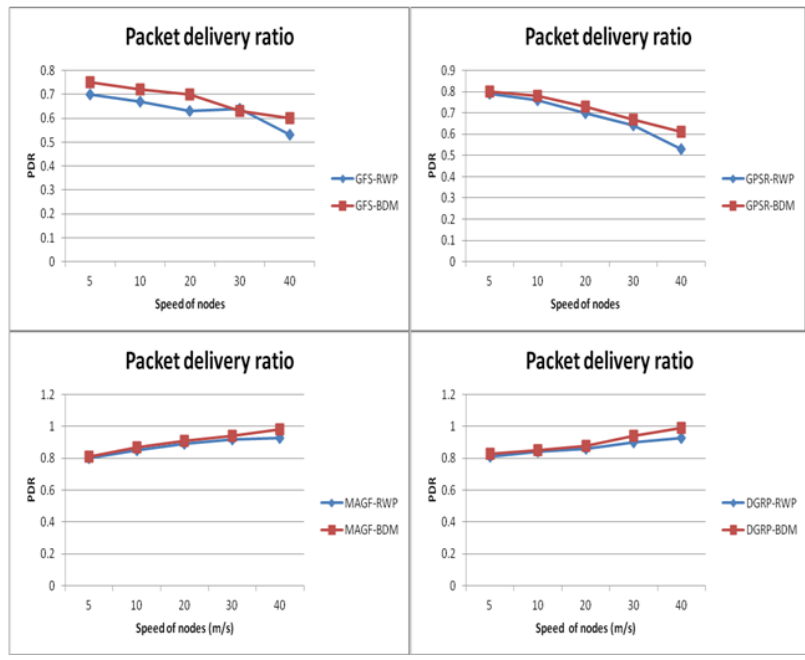

Figs 13-16: Packet delivery ration with the selected routing protocols vs. both mobility models using various speed values

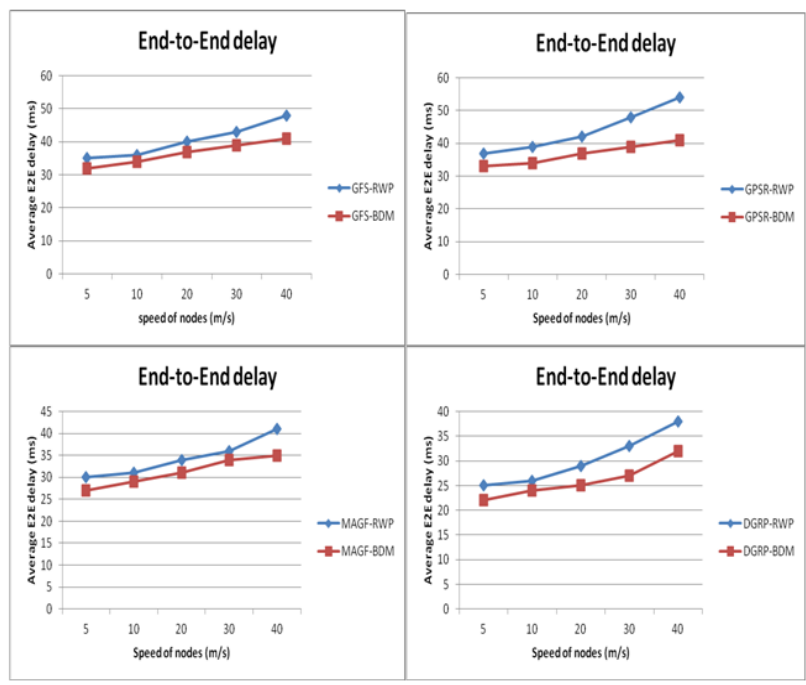

Figs 17-20: End-to-end delay ration with the selected routing protocols vs. both mobility models using various speed values

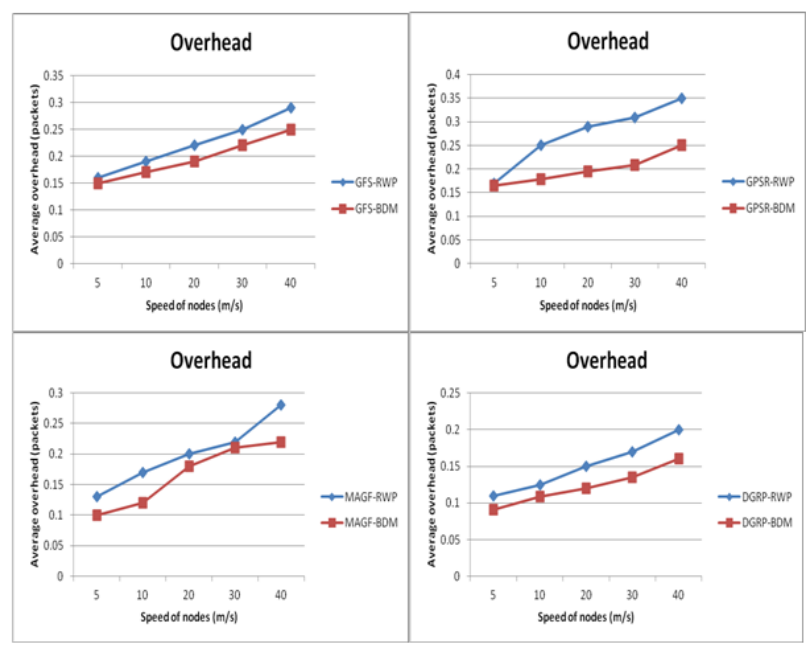

Figs 21-24: Overhead ration with the selected routing protocols vs. both mobility models using various speed values 
For RWP as speed is increasing, the delivery ratio decreases. The situation is also the same with BDM, but with higher delivery ratio compared to RWP. The results for RWP and $\mathrm{BDM}$ are explained by the fact that the inaccuracy of location information increase as the speed of MNs increase which result in packet loss. Both of RWP and BDM showed that the routing delivery ratio is inversely proportional to the increment speed value of nodes.

The high latency value in MNs using RWP refers to the distribution nature at the center of simulated area which introduces congestion and cause packet drop and retransmission and rerouting. May, at the same time the low latency value for MNs using BDM can be attributed to the congestion scarcity to be occurred. With both mobility models, the common tendency is that the end-to-end delay is directly proportional with the increment speed value of nodes.

The increasing of overhead value with RWP is referring to the movement changes in direction with sudden stops and sharp turns of each MN. When the speed of MNs is increasing, the topology will change frequently; this will result in increasing the number of control packets required to update the location information, thus overhead increases too. Also what we noticed here is that the routing overhead is directly proportional to node speed with both mobility models.

\section{CONCLUSION AND FUTURE WORK}

This work is focused on the performance of GPSR with selected performance metric in terms of two mobility models. To perform the performance evaluation process, a detail simulation was executed using the network simulator Ns 2.33. The simulation results with the two conducted scenarios have shown that Boundless mobility model achieved a better system performance than Random Waypoint mobility model. The results indicate that Boundless mobility model produces the highest delivery ratio, lowest end-to-end delay and control overhead compared to Random Waypoint mobility model. Also, the cropped result prove that the performance of GPSR position-based routing protocol varied across different parameters used in the two scenarios through varying the number of nodes and the values of speed. Further study should be assigned to the Boundless mobility model on the real implementation to be sure if it is suitable to be deployed in real-life implementation.

\section{REFERENCES}

[1] P. Ghosekar, et al., "Mobile Ad hoc Networking: Imperatives and Challenges," International Journal of Computer Applications IJCA, vol. 1, pp. 153-158, 2010.

[2] A. Ambhaikar and L. Sharma, "Exploring the Behavior of Mobile Ad Hoc Network Routing Protocols with Reference to Speed and Terrain Range," in Proc. of the IMECS10, 2010, pp. 798-803.

[3] C. Bettstetter, "Mobility Modeling in Wireless Networks: Categorization, Smooth Movement, and Border Effects," ACM SIGMOBILE Mobile Computing and Communications Review, vol. 5, pp. 55-67, 2001.

[4] T. Camp, et al., "A Survey of Mobility Models for Ad Hoc Network Research," Wireless communications and mobile computing, vol. 2, pp. 483-502, 2002.
[5] F. Bai, N. Sadagopan, and A. Helmy, "Important: A framework to systematically analyze the impact of mobility on performance of routing protocols for ad hoc networks,' in the Proc. of the IEEE INFOCOM, 2003, pp. 825-835.

[6] S. Kurkowski, et al., "Constructing MANET Simulation Scenarios that Meet Standards," in Proc. of the MASS 007, 2007, pp. 1-9.

[7] G. G. Finn, "Routing and Addressing Problems in Large Metropolitan-Scale Internetworks," University of Southern California Marina Del Rey Information Sciences InstISI Research Report, ISU/RR-87-180, March 1987.

[8] B. Karp and H. T. Kung, "GPSR: greedy perimeter stateless routing for wireless networks," in Proc. of the sixth annual ACM MobiCom 00, 2000, pp. 243-254.

[9] R. Kumar and S. Rao, "Directional Greedy Routing Protocol (DGRP) in Mobile Ad-Hoc Networks," in Information Technology, ICIT, International Conference on, 2008, pp. 183-188.

[10] J. Li and S. M. Shatz, "Toward using node mobility to enhance Greedy-forwarding in geographic routing for mobile ad hoc networks," in Proc. MODUS008, 2008, pp. 1-8.

[11] D. B. Johnson and D. A. Maltz, "Dynamic Source Routing in Ad hoc Wireless Networks," in Mobile Computing, vol. 353, 1996.

[12] Z. J. Haas, "A New Routing Protocol for the Reconfigurable Wireless Networks," In Proc of the ICUPC, 1997, pp. 562-566.

[13] C. H. Chou, et al., "Dynamic route maintenance for geographic forwarding in mobile ad hoc networks," Computer Networks, vol. 52, pp. 418-431, 2008.

[14] B. Divecha, et al., "Impact of Node Mobility on MANET Routing Protocols Models," Journal of Digital Information Management, vol. 5, p. 19, 2007.

[15] J. Härri, et al., "Vehicular Mobility Simulation with VANET Mobi-Sim," Transactions of the Society for Modeling and Simulation, vol. 87, p. 275, 2011.

[16] G. Yan, et al., "The Role of Network and Mobility Simulators in Evaluating Vehicular Networks," in Intelligent Computing and Information Science, pp. 706712, 2011.

[17] "The Network Simulator - $\quad$ ns-2," http://www.isi.edu/nsnam/ns/, 2002.

[18] C. Bettstetter, "Mobility Modeling in Wireless Networks: Categorization, Smooth Movement, and Boder Effects," ACM Mobile Computing and Communications Review (SIGMOBILE), vol. 5, no. 3, pp. 55-67, Jul. 2001 [Online]. http://doi.acm.org/10.1145/584051.584056

[19] T. Camp, J. Boleng, and V. Davies, "A Survey of Mobility Models for Ad Hoc Network Research," Wireless Communications \& Mobile Computing (WCMC): Special Issue On Mobile Ad Hoc Networking: Research, Trends And Applications, vol. 2, no. 5, pp. 483-502, Aug. 2002. [Online]. Available: http://dx.doi.org/10.1002/wcm.72 
[20] J. Broch, D. A. Maltz, D. B. Johnson, Y.-C. Hu, and J. Jetcheva, "A Performance Comparison of Multi-Hop Wireless Ad Hoc Network Routing Protocols," in the Preceding of the 4th Annual ACM/IEEE International Conference on Mobile Computing and Networking (MobiCom). Dallas, Texas, USA: ACM, 25-30, Oct. 1998, pp. 85-97. [Online]. Available: http://doi.acm.org/10.1145/288235.288256

[21] A. Jardosh, E. M. Belding-Royer, K. C. Almeroth, and S. Suri, "Towards Realistic Mobility Models for Mobile Ad Hoc Networks," in the Preceding of the 9th Annual International Conference on Mobile Computing and Networking, (MobiCom). San Diego, CA, USA: ACM, 14-19, Sep. 2003, pp.217-229. [Online]. Available: http://doi.acm.org/10.1145/938985.939008

[22] F. Bai, N. Sadagopan, and A. Helmy, "The IMPORTANT Framework for Analyzing the Impact of
Mobility on Performance of Routing for Ad Hoc Networks," AdHoc Networks Journal, vol. 1, no. 1, pp. 383-403, Nov. 2003. [Online]. Available: http://dx.doi.org/10.1016/S1570-8705(03)00040-4

[23] A. Chaintreau, P. Hui, J. Crowcroft, C. Diot, R. Gass, and J. Scott, "Impact of Human Mobility on the Design of Opportunistic Forwarding Algorithms," in the Preceding of the.25th IEEE International Conference on Computer Communications, Barcelona, Catalunya, Spai, 23-29, Apr. 2006, pp. 1-13. [Online]. Available: http://dx.doi.org/10.1109/INFOCOM.2006.172

[24] M. Grossglauser and D. Tse, "Mobility Increases the Capacity of Ad Hoc Wireless Networks," IEEE/ACM Transactions on Networking, vol. 10, no. 4, pp. 477-486, Aug. 2002. [Online]. Available: http://dx.doi.org/10.1109/TNET. 2002.801403 